\title{
Aggressions-Assessment im stationären Bereich
}

\author{
Denis Wegmann
}

Auf geschützten Akutstationen liegt der Fokus darauf, Sicherheit für die Hilfesuchenden und das Personal zu gewährleisten. Damit Sie Anzeichen selbst- oder fremdgefährdenden Verhaltens frühzeitig erkennen, stellen wir Ihnen im Interventionskoffer ein AggressionsAssessment vor.

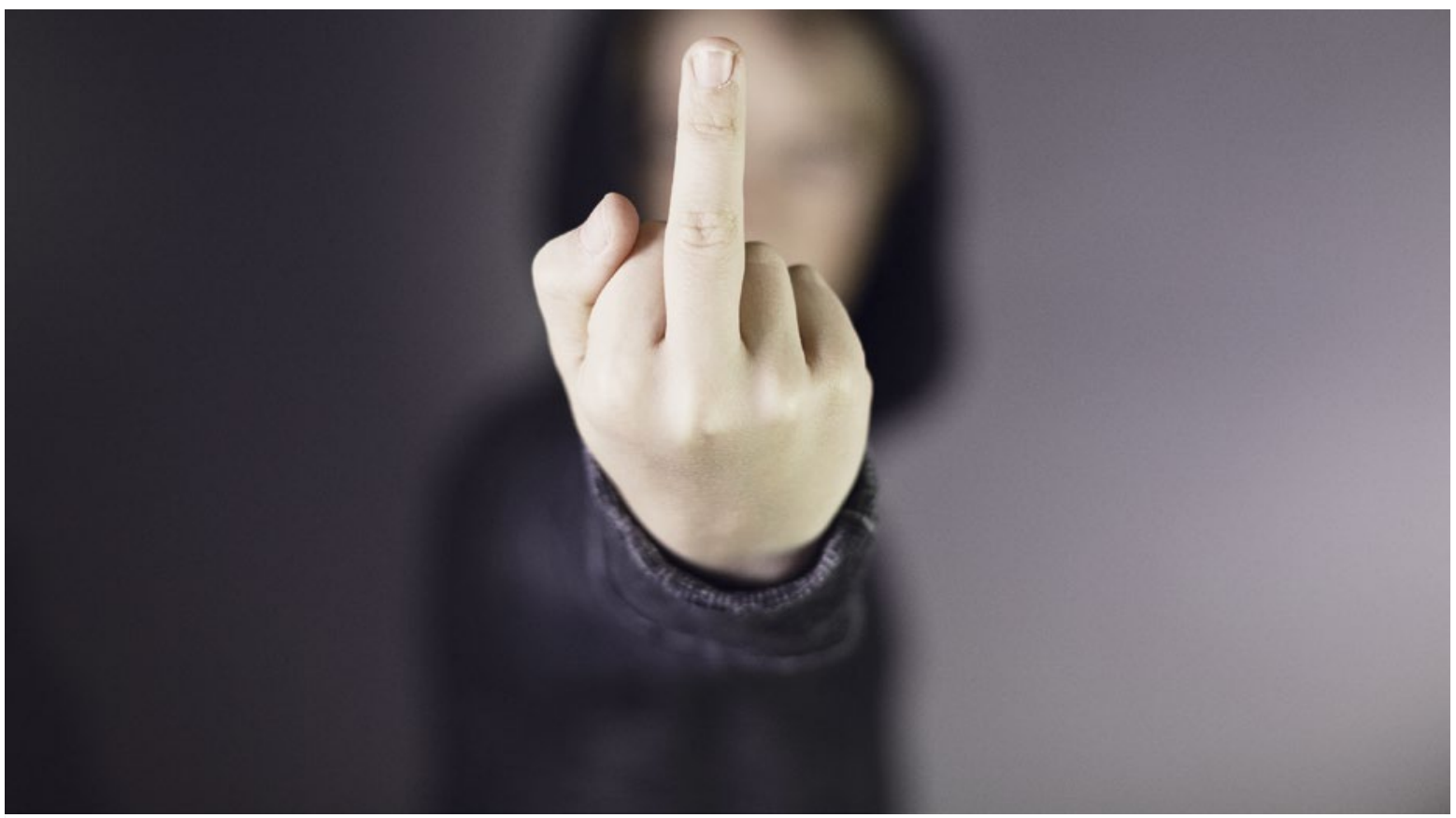

Verhaltensweisen, die vor einem physischen Angriff häufig beobachtet werden, sind verbales und körperliches Drohen oder ... (Symbolbild/Quelle: Kara_AdobeStock)

In akutpsychiatrischen Einrichtungen ist es eines der Hauptziele, eine sichere Behandlung zu gewährleisten. Betroffen sind Klienten, die sich in einer selbst- oder fremdgefährdenden Krise befinden oder deren Wahrnehmung durch eine bestehende psychische Krankheit beeinträchtigt ist. Um die Anzeichen gefährdenden Verhaltens frühzeitig zu erkennen, entstand die Idee, ein Aggressions-Assessment im Rahmen meiner Weiterbildung zum Fach-Gesundheits- und Krankenpfleger zu implementieren und zu überprüfen. Dies soll Mitarbeitern eine objektive Einschätzung ermöglichen. Anhand eines Punktesystems können sie das Risiko für aggressives Verhalten einschätzen.

\section{Welche Mitarbeiter sind betroffen?}

Nach einer Studie von Richter und Berger sind vor allem jüngere beziehungsweise berufsunerfahrene Mitarbeiter von gewaltsamen Übergriffen betroffen. Bei Auszubildenden ist das Risiko sogar 2,5-fach erhöht. [1] Verschiedene Studien haben gezeigt, dass

- 50 Prozent der Ärzte, Psychologen und Sozialarbeiter mindestens einmal während der beruflichen Tätigkeit ernsthaft verletzt worden sind. [2]

- 70 Prozent der Pflegekräfte in der Psychiatrie mindestens einmal in ihrer beruflichen Laufbahn tätlich angegriffen worden sind. [3]

- Zehn Prozent der betroffenen Mitarbeiter ärztlich behandelt werden mussten. [1] 


\section{PILOTSTATION}

Die ausgewählte Station ist eine psychiatrische Intensivstation zur Krisenintervention für Erwachsene im Pfalzklinikum in Klingenmünster. Sie bietet Hilfe und Schutz in schweren seelischen Krisen oder bei akuten psychischen Erkrankungen, speziell wenn Betroffene oder auch Dritte gefährdet sind. Hauptziel des Behandlungsangebots ist es, die Betroffenen dabei zu unterstützen, so schnell wie möglich wieder mehr Verantwortung für sich übernehmen zu können. Das Behandlungskonzept verbindet eine persönliche, von Wertschätzung und Respekt geprägte, multiprofessionelle Intensivbetreuung mit Akutinterventionen in Form von lebenspraktischen Hilfen, psychotherapeutischen Gesprächsangeboten und Medikamenten.

In fünf Prozent der Fälle waren die Mitarbeiter arbeitsunfähig.

\section{Ziel: Objektive Einschätzung des Klienten}

Ziel des Projekts war es, eine objektive Einschätzung des Aggressionsniveaus eines Klienten zu zwei verschiedenen Zeitpunkten zu geben. Das hatte im weiteren Verlauf zum Ziel, frühzeitig einem physischen Aggressionsverhalten deeskalierend entgegenwirken zu können, um somit Schaden vom Betroffenen und seinen Interaktionspartnern abzuwenden. Darüber hinaus war auch von Interesse, objektive und subjektive Einschätzungen, die getrennt voneinander durchgeführt wurden, miteinander zu vergleichen. Es wurde nicht erhoben, wie häufig auf der Pilotstation aggressive Übergriffe von Patienten vorkamen, die im Vorfeld nicht erkannt wurden und ob sich die Anzahl der Übergriffe nach Einführung verändert. Grundsätzliche Ziele waren die Implementierung des Instruments und die Überprüfung und Bestätigung der Checkliste. Die Forschungsfragen lauteten:

- Kann das Assessment-Instrument die Messung des Aggressionsniveaus auf einer akutpsychiatrischen Station unterstützen?

- Stimmt die Einschätzung des Aggressionsniveaus der Brøset-Skala ( $\triangleright$ Abb. 1) mit der subjektiven Wahrnehmung des Beurteilers überein?

\section{Effektive Unterstützung des Teams}

Ziel: In einem Projektteam sollte ein Assessment mit seinen Rahmenbedingungen festgelegt, anschließend im Team implementiert und im Klientendokumentationssystem „Medico/S“ dokumentiert werden.
DIE NORWEGISCHE BRøSET-GEWALTCHECKLISTE ( $\triangleright$ ABB. 1)

Die Brøset-Gewalt-Checkliste (Brøset Violence Checklist, BVC) wurde von dem Pflegeforscher Dr. Roger Almvik in Trondheim/Norwegen entwickelt. Sie beruht auf einer ebenfalls norwegischen Untersuchung von Linaker und Busch-Iverson über Verhaltensweisen, die in den 24 Stunden vor einem physischen Angriff häufig beobachtet werden. Die Brøset-Gewalt-Checkliste ist ein Instrument, mit dem das Risiko eingeschätzt werden kann, ob ein akutpsychiatrischer Patient in den 8-12 Stunden nach der Einschätzung jemanden physisch angreifen wird. Die Checkliste umfasst 6 Verhaltensweisen, bei denen 2-mal täglich eingeschätzt wird, ob sie in den letzten Stunden beziehungsweise seit der letzten Einschätzung beobachtet worden sind (zum Beispiel um 9 Uhr und um 18 Uhr). Diese Verhaltensweisen sind:

- Verwirrtheit,

- Reizbarkeit,

- lärmiges Verhalten,

- verbales Drohen,

- körperliches Drohen,

- Angriffe auf Gegenstände.

Jede Verhaltensweise wird 2-mal täglich als „beobachtet“ mit 1 Punkt oder als „nicht beobachtet“ mit 0 Punkten bewertet.

Die Summe (0-6 Punkte) entspricht der Wahrscheinlichkeit eines physischen Angriffs in den Stunden bis zur nächsten Einschätzung. 3 oder mehr Punkte gelten als hohes Risiko und der Einsatz präventiver Maßnahmen sollte geplant werden. [4] In der norwegischen Originalstudie erwies sich das Instrument als gut in den Arbeitsalltag integrierbar, einfach anzuwenden und es wurde eine befriedigende Interrater-Reliabilität festgestellt. [5] Die Sensitivität des Instruments betrug 0.74 , die Spezifität 0.91, die ROC-Analyse ergab eine Fläche unter der Kurve (Area under the curve, AUCROC) von 0.82 (95 Prozent $\mathrm{Cl} 0.75$ - 0.89). Diese Werte konnten in einer schweizerischen Studie mit einer deutschen Übersetzung bestätigt werden. [6]

\section{Wie wird die BVC gewöhnlich eingesetzt?}

Routinemäßig erfolgt die Risikoeinschätzung bei allen Aufnahmen:

- direkt bei der Aufnahme,

- am Aufnahmetag

- und an den folgenden 3 Tagen;

- nach Ablauf der ersten 3 Tage erfolgen weitere Einschätzungen, wenn die Bezugsperson und/ oder das Team dies angezeigt findet. [7] 


\section{Wer nimmt die Risikoeinschätzung vor?}

Die Einschätzung wird von einer Pflegeperson vorgenommen, die über das Verhalten des Patienten in der Beurteilungsperiode informiert ist, durch eigene Beobachtungen/Kontakte und/oder durch Mitteilungen von Kollegen (im Bedarfsfall nachfragen). Auf vielen Stationen wird die Einschätzung einem bestimmten Dienst (zum Beispiel Frühdienst) zugeordnet. Die Einschätzung kann auch gemeinsam von mehreren Personen vorgenommen werden. Bei Schichtbetrieb erfolgt die Einschätzung am besten etwa zwei Stunden nach Beginn einer Schicht.

\section{Was ist der Beurteilungszeitraum?}

Die 6 Verhaltensweisen werden mit „1“ bewertet, wenn sie seit der letzten Einschätzung beobachtet wurden, mit „0“, wenn sie seit der letzten Einschätzung nicht beobachtet wurden.
Zunächst nahm ich an der Fachtagung „Autonomie und Selbstbestimmung in der Psychiatrie“ in Düsseldorf teil. Hier wurden die aktuellen wissenschaftlichen Ergebnisse zur klinischen Aggressionsprävention aus ärztlicher und pflegerischer Sicht diskutiert. Bei weiteren Recherchen stieß ich auf die Brøset-Skala, die mir sehr gut geeignet schien, die geforderte objektive Einschätzung des Aggressionsniveaus darzustellen. Damit stand mir ein validiertes, in anderen Kliniken bereits gebräuchliches Instrument zur Verfügung. Im Laufe meines Projekts fand ich es interessant, diese objektive Einschätzung mit dem subjektiven Eindruck, den die Teammitglieder vom Aggressionsniveau der beurteilten Klienten hatten, zu vergleichen.

Die Umsetzung der Datenerhebungsphase des Projekts wurde mit den verantwortlichen Leitungen der Pilotstation und den Projektteammitgliedern ausführlich diskutiert. Wir vereinbarten, dass bei jedem aufgenommenen Klienten eine erste subjektive Einschätzung in „nicht“, „leicht“, „mäßig“ oder „sehr aggressiv“ schon während der administrativen Aufnahme durch die Pflege auf Station erfolgen

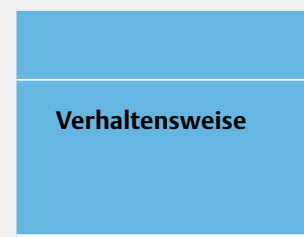

Verwirrt

Reizbar

Lärmig

Körperliches Drohen

Verbales Drohen

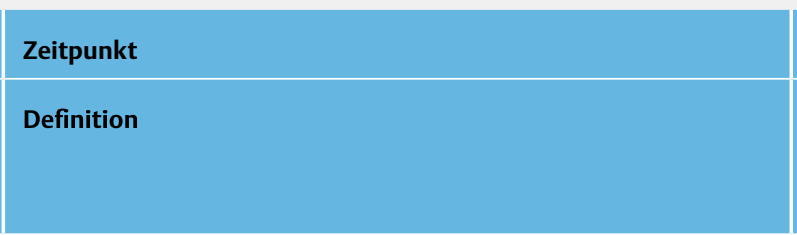

Erscheint offensichtlich verwirrt und desorientiert. Ist sich möglicherweise der Zeit, des Ortes und der Personen nicht bewusst; verkennt Personen, Situationen. „Verwirrt“ ist in einem allgemeinen Sinn gemeint. Hierzu zählt auch psychotische Verwirrtheit, nicht nur Verwirrtheit in Zusammenhang mit einer Demenz o. ä.

Ist schnell verärgert oder wütend; zum Beispiel nicht in der Lage, die Anwesenheit anderer zu tolerieren.

Das Verhalten ist übermäßig laut oder Krach verursachend. Z. B. schägt Türen, schreit beim Sprechen, etc.

Eine deutliche Absicht, eine andere Person zu bedrohnen. Z. B. eine aggressive Körperhaltung einnehmen, an der Kleidung einer anderen Person reißen, Ballen der Faust, Heben eines Armes oder Fußes.

Ein verbaler Ausbruch, der mehr ist als nur eine erhobene Stimme; und der die klare Absicht hat, eine andere Person zu verängstigen/ einzuschüchtern, z. B. verbale Angriffe, Beschimpfungen, verbal neutrale Kommentare, die auf eine knurrende aggressive Art und Weise geäußert werden.

Angriff auf Eine aggressive Handlung, die sich gegen einen Gegenstand und Gegenstände nicht gegen eine Person richtet, z. B. das wahllose Zuschlagen oder Zerschlagen von Fenstern, Treten, Schlagen oder Kopframmen gegen einen Gegenstand, oder Zerschlagen von Möbeln.

Summe = Risiko für physischen Angriff in der nächsten Schicht

Interpretation

0 Punkte $=$ geringes Risiko

$1-2$ Punkte = mäßiges Risiko, es sollten präventive Massnahmen ergriffen werden
$\geq 3$ Punkte = hohes Risiko, es sind präventive Massnahmen und Pläne zum Umgang mit einem allfäl-
ligen Angriff erforderlich

$1-2$ Punkte = mäßiges Risiko, es sollten präventive Massnahmen ergriffen werden
$\geq 3$ Punkte = hohes Risiko, es sind präventive Massnahmen und Pläne zum Umgang mit einem allfäl-
ligen Angriff erforderlich

$1-2$ Punkte = mäßiges Risiko, es sollten präventive Massnahmen ergriffen werden
$\geq 3$ Punkte = hohes Risiko, es sind präventive Massnahmen und Pläne zum Umgang mit einem allfäl-
ligen Angriff erforderlich

\begin{tabular}{|l|l|}
\hline Vormittag & Abend \\
\hline $\begin{array}{l}\text { Punkte } \\
\text { (beobachtet }=\end{array}$ & $\begin{array}{l}\text { Punkte } \\
\text { (beobachtet }=\end{array}$ \\
1 Punkt) & 1 Punkt)
\end{tabular}

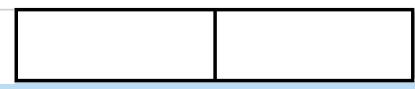

Abb. 1 Norwegische Brøset-Gewalt-Checkliste. (Quelle: Almvik R. The Brøset Violence Checklist (BVC). Im Internet: http:// riskassessment.no/ (files/BVC-English-version.pdf)) 


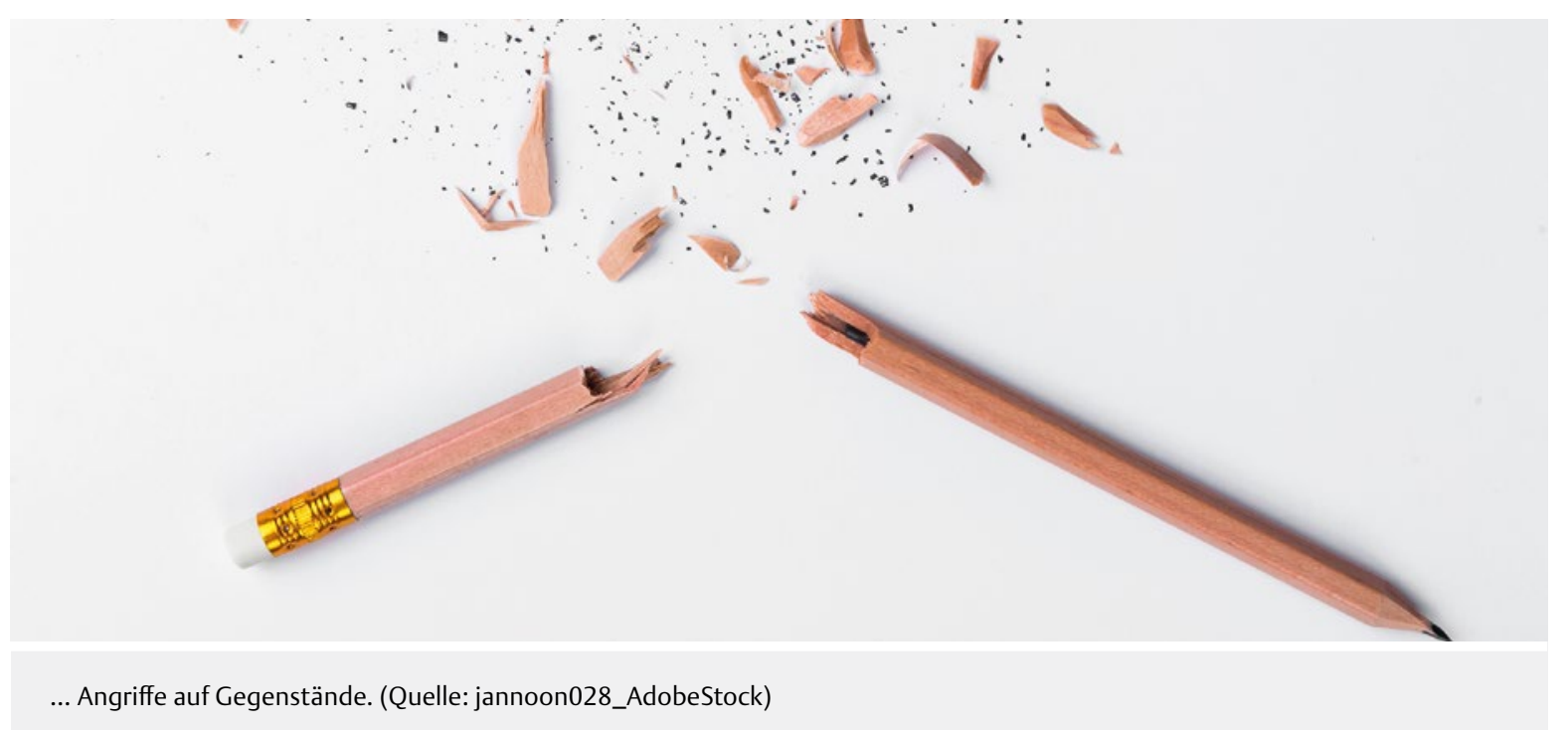

soll. Direkt danach und nach acht Stunden, als Verlaufskontrolle, kam die Brøset-Skala zum Einsatz. Diese Ergebnisse wurden in der Klientenakte dokumentiert. Zur Sicherstellung der Datengüte wurde das Zweit-Assessment (nach acht Stunden) durch Terminierung im Outlook unterstützt.

Nachdem diese Rahmenbedingungen erarbeitet waren, realisierte die EDV-Abteilung des Hauses in enger Zusammenarbeit eine anwenderfreundliche EDV-gestützte Erfassung des Aggressions-Assessments. Nach einer ersten Erprobungsphase des EDV-gestützten Instruments auf einer virtuellen Teststation erfolgten weitere Modifikationen, die eine Nutzung erleichterten. So wurden durch Mausklick Begriffserläuterungen angezeigt, das Abspeichern des Assessment-Bogens vereinfacht und eine Verknüpfung mit der aktuellen Verlaufsdokumentation des Klienten ermöglicht. Dadurch war für alle am Therapieprozess Beteiligten das Assessment-Ergebnis sichtbar.

Das Projekt und das optimierte Assessment-Instrument wurden im Rahmen eines Strukturteams dem Stationsteam der Pilotstation vorgestellt. Hierzu entwarf ich einen Workflow, welcher zum besseren Verständnis der Durchführung diente. Nach dem Start der Pilotphase konnte ich feststellen, dass das Assessment konsequent bei jedem aufgenommenen Klienten angewendet wurde. Eine weitere Motivation des Teams zur Annahme der Methode war nicht notwendig. In ersten Rückmeldungen im Rahmen des Projekts werteten die Teammitglieder die Brøset-Skala als effektive Unterstützung zur Einschätzung des Erregungsniveaus, besonders bei unbekannten Klienten.

\section{Evaluation}

Das implementierte Aggressions-Assessment, das zum Zeitpunkt der Aufnahme und wiederholt acht Stunden danach das Erregungsniveau eines Klienten einschätzt, wurde evaluiert. Hierzu wurde eine Stichprobe genommen und der Zeitraum auf ein halbes Jahr begrenzt. In jedem
Eintrag ist eine subjektive Einschätzung vorhanden, die sich nach dem subjektiven Empfinden der Fachkraft richtet und sich in „nicht“, „leicht“, „mäßig“ oder „sehr gewaltbereit" unterscheidet. Ebenso errechnet das Instrument einen Score, der sich aus sechs einzuschätzenden Items zusammensetzt. Dieser ergibt entweder ein „geringes“, „mäßiges“ oder „hohes Risiko“ eines physischen Angriffs eines Klienten in der nächsten Schicht. Hierbei wird zur Evaluation nochmals das hohe Risiko anhand der unterschiedlich errechneten Werte differenziert in „hohes“ und „sehr hohes Risiko“.

\section{Ergebnisse}

Aus der Stichprobe ergaben sich 232 angelegte Einschätzungen. Diese klassifizieren das Empfinden der Fachkräfte, die den Großteil der aufgenommenen Klienten als nicht oder wenig aggressiv erleben ( $\triangleright \mathbf{A b b} \mathbf{2} \mathbf{2 a})$.

Durch das Assessment wird anhand der Items ein größerer Teil der Klienten über dem empfundenen Niveau eingeschätzt und ein mäßiges Risiko errechnet ( $\mathbf{A} \mathbf{b b}$. 2b).

Nach der Auswahl der Toleranz der Zweiteinschätzung zur Ersteinschätzung und der Berücksichtigung, dass keine Ersteinschätzungen vorhanden sind, ergab sich ein Datensatz, der 122 Einträge umfasst. Darin bleibt die Verteilung in etwa gleich ( $\triangleright$ Abb. $\mathbf{3}$ ).

Betrachtet man die Einschätzungen der Geschlechter, ist die Wahrnehmung einer Bedrohung durch Männer deutlich stärker als das errechnete Risiko ( $\mathbf{A b b}$.4a). Bei den objektiven Werten verlaufen die Einschätzungen fast parallel ( $>$ Abb. $4 \mathbf{b})$.

Der Vergleich der subjektiven Einschätzung mit der objektiven Einschätzung beweist eine Übereinstimmung der Wahrnehmung mit den ermittelten Werten des Instruments von 60 Prozent ( $\mathbf{A}$ Abb. 5). 


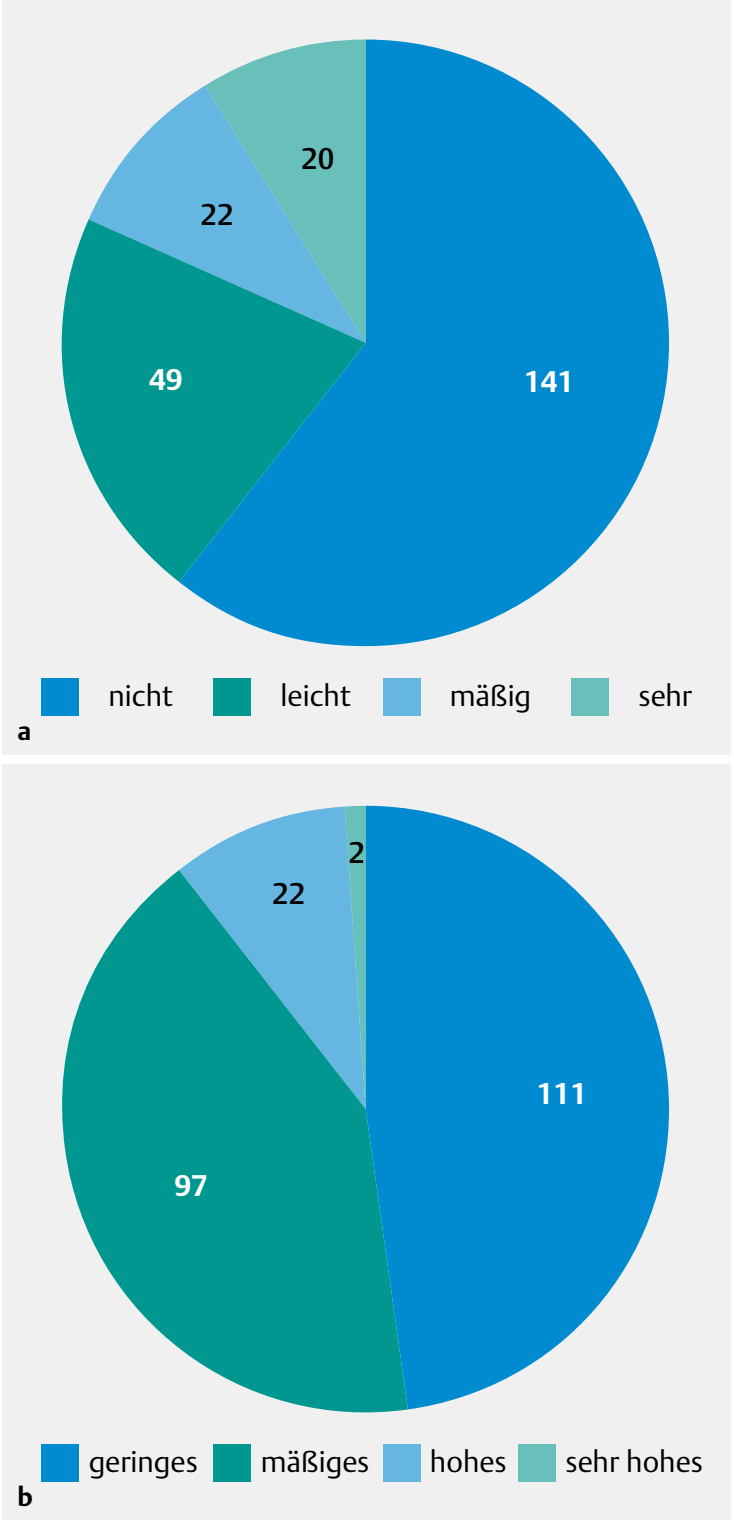

- Abb. 2 a Subjektive und b objektive Einschätzungen des Aggressionsniveaus.

Höher schätzt das Assessment den Erregungszustand eines Klienten zu 16 Prozent und niedriger zu 24 Prozent ein. Die Aufteilung in die einzelnen subjektiven Sparten lässt erkennen, dass das Assessment bei weniger aggressiv wahrgenommenen Klienten ein höheres Risiko und bei aggressiv wirkenden Klienten ein niedrigeres Risiko einschätzt, es jedoch trotzdem erkennt.

\section{„Vorhersagen sind immer schwierig, besonders wenn sie die Zukunft betreffen."}

Niels Bohr, 1885-1962, dänischer Physiker

Die Auswertung der Daten ergab, dass ein Großteil (62 Prozent) der Klienten, bei denen das Formular „Freiheitsent-

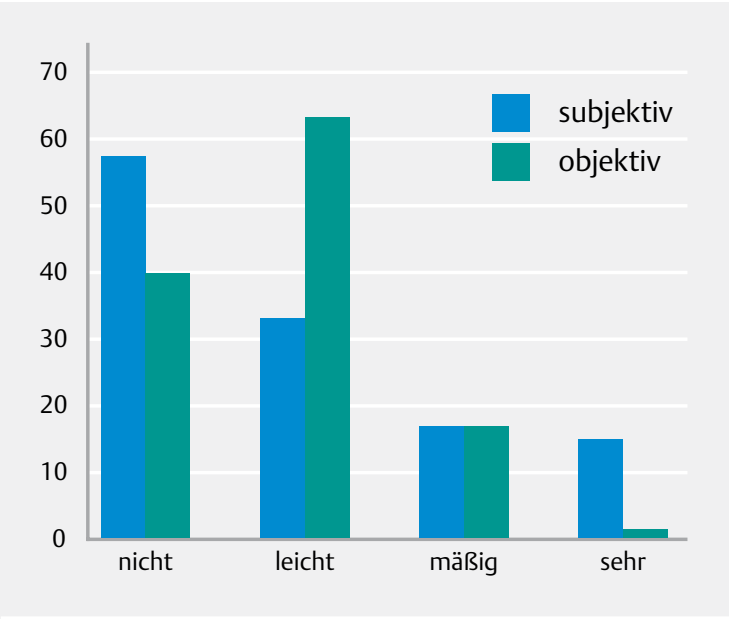

- Abb. 3 Vergleich der Auszählung nach Selektion.

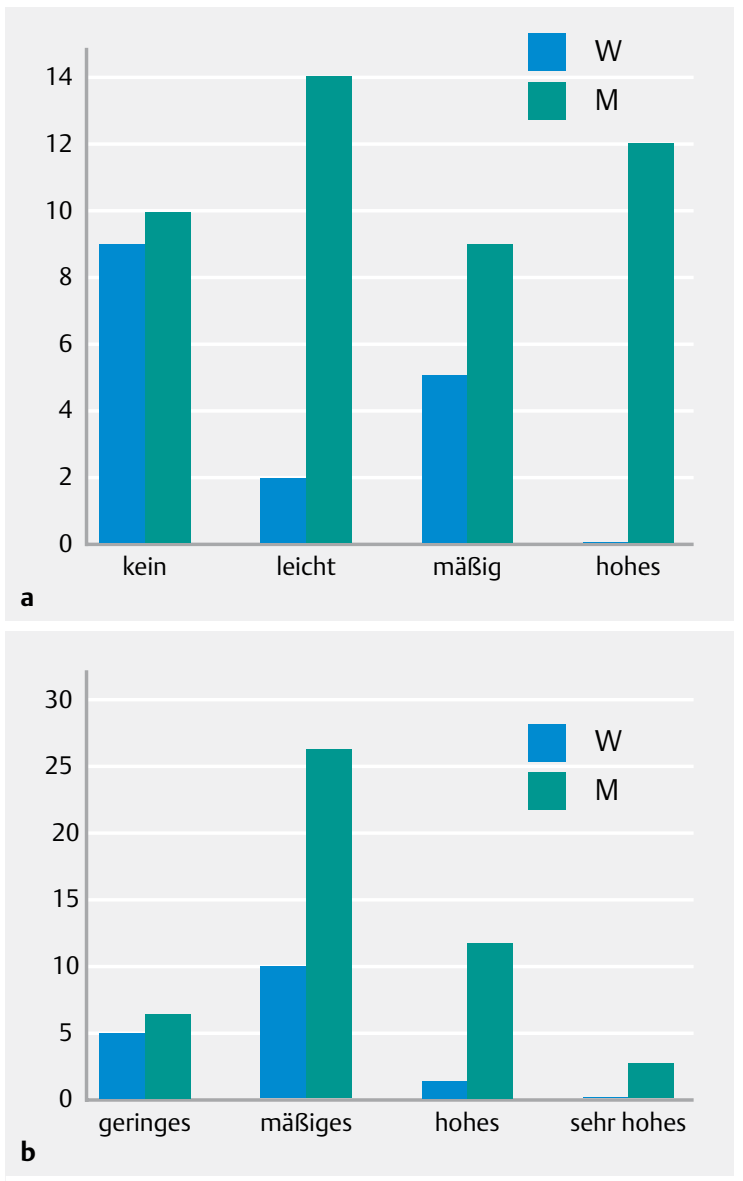

- Abb. 4 a Subjektives und b objektives Aggressionsniveau.

ziehende Maßnahme“ im besagten Zeitraum vorhanden ist, fixiert werden musste. Zwar mussten bei den anderen 38 Prozent auch Zwangsmaßnahmen angewendet werden, jedoch weniger intensive und traumatisierende, wie zum Beispiel Zwangsmedikation (33 Prozent) oder räumliche Begrenzung im Sinne einer Zimmer-Isolation (fünf Prozent). 


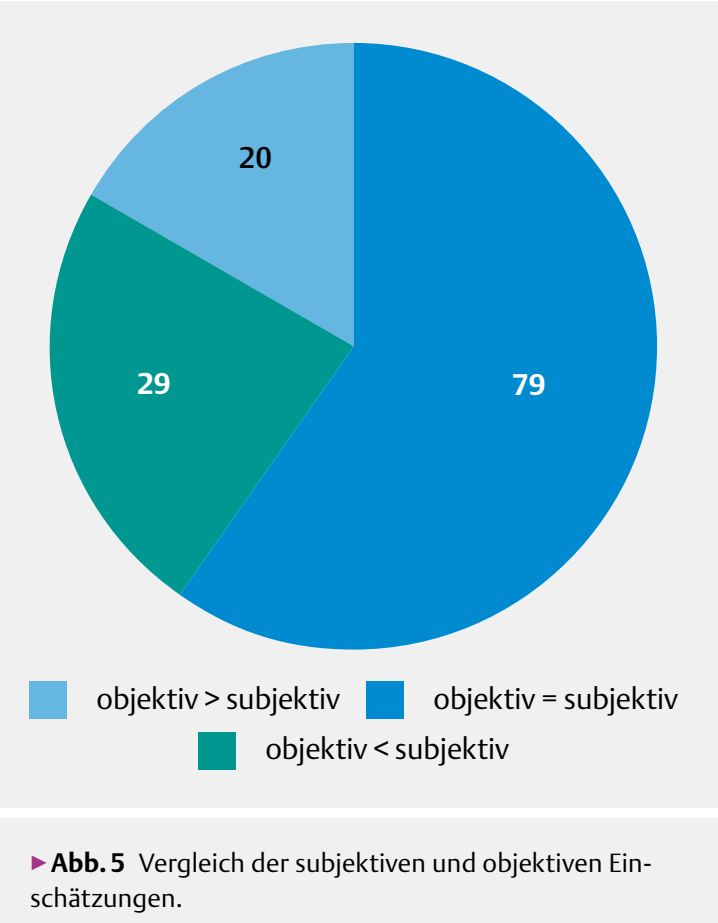

schätzungen.

\section{FAZIT}

Das Aggressions-Assessment misst anhand der sechs objektiven Items das Erregungsniveau eines Klienten zuverlässig und schätzt es als unterschiedliches Risiko ein. Die subjektiven Einschätzungen stimmen zum größten Teil mit den objektiv errechneten Werten überein. Da die Ergebnisse der objektiven und subjektiven Einschätzungen sehr ähnlich sind, lässt sich überspitzt fragen, ob dies den Einsatz der Brøset-Gewalt-Checkliste überhaupt rechtfertigt. Tatsächlich dient das Instrument zur Objektivierung des subjektiven Eindrucks. Auch ist es eine gute Unterstützung für unerfahrene Kollegen und Auszubildende, um frühzeitig einem Erregungszustand eines Klienten deeskalierend entgegenwirken zu können und somit Schaden vom Betroffenen und seinen Interaktionspartnern abzuwenden.

Die Ergebnisse aus der vorliegenden Stichprobe sind ausschließlich auf das Funktionieren der Checkliste bezogen. Es ging nicht darum, festzustellen, wie häufig auf der Pilotstation aggressive Übergriffe von Patienten vorkamen und ob sich durch den Einsatz des Instruments die Anzahl der Übergriffe verändert hat. Eine Intervention nach einem Standard ist ebenso nicht das Ziel gewesen. Bei Krisen sollten weiterhin individuelle Lösungsstrategien erarbeitet und keinesfalls anhand eines Handlungskatalogs deeskaliert werden.

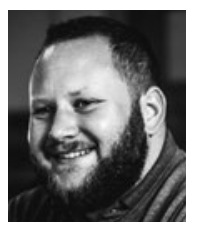

\section{Denis Wegmann}

Fach-Gesundheits- und Krankenpfleger, Forensisch-Psychiatrische Ambulanz, Klinik für Forensische Psychiatrie, Pfalzklinikum für Psychiatrie und Neurologie AdöR

E-Mail: denis.wegmann@pfalzklinikum.de

\section{Literatur}

[1] Richter D, Berger K. Patientenübergriffe auf Mitarbeiter - Eine prospektive Untersuchung der Häufigkeit, Situationen und Folgen. Nervenarzt 2001; 72: 693-699

[2] Steinert T. Aggression bei psychisch Kranken. Stuttgart: Enke; 1995

[3] Abderhalden C et al, 2002. In: Leupold J, Dietrichs S, Baumgart I et al. Pflegekonzept: Gewalt und Aggressionen in der psychiatrischen Pflege. Universitätsmedizin Mainz: Projektarbeit in der Fachweiterbildung 2012-2014. Im Internet: http://www.unimedizin-mainz.de/ (typo3temp/ secure_downloads/7380/0/7b937c785d51d2bda6bdc3b95a64510dba268967/Pflegekonzept___Gewalt_und_ Aggressionen_in_der_psychiatrischen_Pflege.pdf); Stand: 17.04.2019

[4] Linaker OM, Busch-Iversen H. Predictors of imminent violence in psychiatric inpatients. Acta Psychiatrica Scandinavica 1995; 92: 250-254

[5] Almvik R, Woods P. The Broset Violence Checklist (BVC) and the prediction of inpatient violence: Some preliminary results. Perspectives in Psychiatric Care 1998; 5: 208-211

[6] Almvik R, Woods P, Rasmussen K. The Broset Violence Checklist: Sensitivity, Specificity and Interrater Reliability. Journal of Interpersonal Violence 2000; 15: 1284-1296

[7] Almvik R, Woods P. Short-term risk prediction: the Broset Violence Checklist. Journal of Psychiatric and Mental Health Nursing 2003; 10: 236-238

[8] Almvik R. The Brøset Violence Checklist (BVC ${ }^{\odot}$ ). Im Internet: http://riskassessment.no/ (files/BVC-English-version.pdf); Stand: 17.04 .2019

Bibliografie

DOI https://doi.org/10.1055/a-0864-7347

Psych. Pflege Heute 2019; 25: 110-115

(C) Georg Thieme Verlag KG Stuttgart · New York ISSN 0949-1619 